\title{
The Effect of Low Dose Aspirin on Placental Vascular Thrombosis in Pregnancies Complicated by Lupus Anticoagulant and Anticardiolipin Antibodies
}

\author{
Hamid A. Hadi, M.D., Susan Parks, M.D., James Finley, M.D. \\ Greenville, NC
}

DOI: http://dx.doi.org/10.5915/22-4-14315

\begin{abstract}
We followed six pregnant women with a history of pregnancy losses and presence of anticardiolipin antibodies. Two of these patients were also positive for lupus anticoagulant antibody. All patients were placed on low dose aspirin ( $81 \mathrm{mg} /$ day) from the first trimester onward. All women were delivered between 30-40 weeks of pregnancy because of evidence of intrauterine growth retardation and abnormal fetal surveillance tests. Microscopic examination of the placentas showed thrombi involving large and medium sized vessels in tertiary stem villi and prominent medial hyperplasia in small and medium sized arterioles. This study suggests that low dose aspirin therapy may ameliorate, but does not prevent, formation of placental vascular thrombi.
\end{abstract}

Key words: Aspirin, lupus anticoagulant/anticardiolipin antibodies, placental vascular thrombosis.

Lupus anticoagulant and anticardiolipin antibodies (usually IgG, rarely IgM) have been associated with decidual and vascular thrombosis, placental infarction and fetal wastage..$^{1,2}$ The mechanism of thrombosis in these patients is unknown. The damage of phospholipid-rich platelets by these antibodies and release of thromboxane $A_{2}$, coupled with impairment of prostacyclin production by vascular endothelial cells constitute low dose aspirin to reduce the risk of thromobis. Subsequent morbidity and mortality in such circumstances has been suggested but not adequately studied. ${ }^{3}$ In this study, we identified pregnant patients with positive lupus anticoagulant and/or anticardiolipin an-

From the Department of Obstetrics and Gynecology

Division of Maternal-Fetal Medicine and the Department of Pathology

East Caroline University School of Medicine

Greenville, NC

Reprint Requests: Hamid Hadi, M.D.

Department $O B / G Y N$, Maternal-Fetal Medicine

East Carolina University School of Medicine

Greenville, NC 27834 tibodies. Placental pathological findings and fetal outcome are presented.

\section{Materials and methods}

During a two-year study period, six patients with a poor obstetric history who were positive for lupus anticoagulant and/or anticardiolipin antibodies were identified before twelve weeks of gestation and followed until the time of delivery. No other cause for the repeated pregnancy losses was identified in any of the patients. All patients tested positive for anticardiolipin antibody. Only two of the six patients were also positive for lupus anticoagulant antibodies. Of these, one patient had a history of discoid lupus; the other had clinical manifestations of systemic lupus erythmatosis (SLE) on the initial visit and was on prednisone $(30 \mathrm{mg} /$ day $)$. She was also on prophylactic heparin therapy $(10,000$ unit/day) because of history of pulmonary embolism.

Activated partial thromboplastin time was used to detect lupus anticoagulant antibodies. Patients' plasma was initially screened by performing a prothrombin time (PT) and a diluted PT (dilutions of thromboplastin reagent) asay. ${ }^{4} \mathrm{~A}$ ratio above 3 was considered abnormal and indicative of lupus-type anticoagulant. An abnormal finding was verified by a 
Table 1. Pertinent Clinical and Laboratory Data of Study Patients.

\begin{tabular}{|c|c|c|c|c|c|c|}
\hline & \multicolumn{6}{|c|}{ Patient Identification } \\
\hline & $A^{*}$ & B & C & D & $\mathbf{E}$ & $\mathbf{F}$ \\
\hline \multicolumn{7}{|l|}{ Obstetric History } \\
\hline 20 wks. loss & 0 & 2 & 1 & 0 & 2 & 2 \\
\hline 20 wks. loss & 2 & 1 & 1 & 1 & 1 & 0 \\
\hline \multicolumn{7}{|l|}{ Maternal Data } \\
\hline Clinical SLE & Yes & Yes & No & No & No & No \\
\hline Lupus Anticoag. & Yes & Yes & No & No & No & No \\
\hline Anticardiolipin & Yes & Yes & Yes & Yes & Yes & Yes \\
\hline ANA & $1 / 2560$ & $1 / 640$ & $1 / 320$ & $1 / 640$ & $1 / 640$ & $1 / 160$ \\
\hline Anti-DNA & Neg & Neg & Neg & Neg & Neg & Neg \\
\hline \multicolumn{7}{|l|}{ Medication this } \\
\hline pregnancy & Asp** & Asp & Asp & Asp & Asp & Asp \\
\hline & Heparin & & & & & \\
\hline & Prednisone & & & & & \\
\hline
\end{tabular}

*Patient A had a history of pulmonary embolism and two fetal demises at 28 and 32 weeks of pregnancy.

**Asp - Aspirin

platelet neutralization assay in which a partial thromboplastin time (PTT) was performed on the patient's plasma, followed by a second PTT on a 1:1 mixture of the patient's plasma and a frozen/thawed platelet preparation.4, A correction of more than five seconds in the PTT by the platelet neutralization procedure was further evidence of the presence of the lupus-type anticoagulant. An enzyme-linked immunosorbent assay for IgG and IgM was used* to measure antibodies binding to the phospholipid cardiolipin based on the Harris study. ${ }^{6}$

All patients were placed on low dose aspirin ( 81 $\mathrm{mg} /$ day) from the first trimester until the time of delivery. As noted above, one patient was already on Prednisone $30 \mathrm{mg} /$ day for SLE and subcutaneous prophylactic heparin $(10,000$ units twice/day) due to a history of pulmonary embolism. Tests of fetal wellbeing consisted of serial non-stress testing (fetal heart rate monitoring) at weekly intervals starting at 28 weeks until 30 weeks, and then twice weekly. Serial weekly testing of the umbilical artery velocimetry waveform was begun at 24 weeks and continued until the time of delivery. Umbilical artery velocity waveform studies were performed using a continuous-wave Doppler device, operating at a transmit-receive frequency of $4 \mathrm{MHz}$, and a model Multigon 500-A spectrum analyzer. The umbilical blood flow velocity during systole and diastole are displayed and a ratio, the systolic/diastolic ratio, was measured as previously reported. ' This ratio is believed to correlate with the placental resistance to blood

*Smith-Kline Bio-Science Laboratories, Tucker, GA. flow.

Placentas were weighed, fixed in $10 \%$ formalin solution and cross sections made avoiding the areas of infarction. The sections were stained by hematoxylin-eosin and were studied for presence of vascular thrombosis. Furthermore, cross sections of the umbilical cord were done and the umbilical vein and arteries examined. Pertinent clinical data such as obstetric history, antibody test results, as well as fetal well-being tests, and information on pregnancy outcome were collected.

\section{Results}

Clinical and laboratory data of patients are shown in Table 1. Patient A had a history of pulmonary embolus and two intrauterine fetal demises at 28 and 32 weeks of gestation, and had a clinical exacerbation of SLE. In addition to prednisone and prophylactic heparin therapy, she was also placed on low-dose aspirin therapy. The remaining five patients were asymptomatic and were placed on low-dose aspirin.

Table 2 describes the results of fetal surveillance tests and pregnancy outcome. Umbilical artery systolic/diastolic ratio became gradually abnormal (higher) as pregnancy progressed from 24 weeks onward, indicating gradually increasing resistance to placental blood flow. However, there were no instances of reversal of the diastolic component (the most severe abnormal finding). Serial ultrasound measurements of the fetuses suggested intrauterine growth retardation of all fetuses during the third trimester of pregnancy. Amniotic fluid volume was diminished ( $2 \mathrm{~cm}$ pocket in vertical and transverse dimensions) in only three patients. Non-stress tests 
Table 2. Results of Fetal Surveillance Tests and Pregnancy Outcome in Study Patients

Patient Identification

\begin{tabular}{llccccc}
\hline & A & B & C & D & E & F \\
\hline $\begin{array}{l}\text { Fetal Surveillance Tests: } \\
\text { - umbilical artery }\end{array}$ & & & & & & \\
$\quad$ flow velocimetry & Abn & Abn & Abn & Abn & Abn & Abn \\
- ultrasound growth & Abn & Abn & Abn & Abn & Abn & Abn \\
- amniotic fluid volume & Abn & Abn & Norm & Norm & Abn & Norm \\
- non-stress test & Abn & Norm & Norm & Norm & Norm & Norm \\
Pregnancy Outcome: & & & & & & \\
- Gestational Age at & & & & & & \\
$\quad$ Delivery (Weeks) & 32 & 30 & 34 & 33 & 31 & 32 \\
- Route of delivery & CS & CS & CS & CS & CS & CS \\
Anticardiolipin & Yes & Yes & Yes & Yes & Yes & Yes \\
- Birth weight (gm) & 1120 & 1010 & 1165 & 1200 & 996 & 1105 \\
- Neonatal RDS & No & Yes & No & Yes & Yes & Yes \\
\hline
\end{tabular}

Abn $=$ Abnormal $\quad$ Norm $=$ Normal

$\mathrm{CS}=$ Cesarean Section

RDS $=$ Respiratory Distress Syndrome

*All neonates survived

remained normal except in one patient (patient $\mathbf{A}$ ) in whom, prior to delivery, the test was interpreted as non-reactive, i.e., there were no fetal heart rate accelerations with fetal movements. Also seen were spontaneous decelerations. A non-reactive non-stress test, especially with spontaneous decelerations, is considered a sign of fetal ill-health.

All patients were delivered between 30-34 weeks of gestation by caesarean section because of evidence of intrauterine growth retardation, unfavorable cervix, and abnormal fetal surveillance tests, and all neonates survived.

Histological examination of the placentas showed various degrees of thrombi involving subamniotic blood vessels as well as large and medium-sized vessels in tertiary stem villi. Figures 1 and 2 represent vascular thrombosis in one of the study patients. Umbilical cord artery showed deposition of connective tissue and vascular sclerosis. Areas of recent infarctions were also constant features of placental pathology.

\section{Discussion}

Lupus anticoagulant and anticardiolipin antibodies that prolong phospholipid-dependent coagulation tests in vitro have been clinically implicated in the formation of placental vascular thrombi, and consequently fetal wastage, i.e., repeated spontaneous abortions, intrauterine fetal growth retardation and fetal death. ${ }^{1}$ The pathophysiologic mechanisms accounting for vascular thrombosis are unclear, and the role of thromboxane release by platelets damaged by lupus anticoagulant remains speculative. Therefore, the management and choice of drug therapy in this condition also remain controversial.

In this study, we treated six patients with low-dose aspirin from the first trimester until delivery. Only one patient with clinical manifestation of SLE was treated with a combination of low-dose aspirin, prednisone, and prophylactic heparin due to previous pulmonary embolism. All fetuses were growth retarded; all delivered between $30-34$ weeks and survived.

Lube and Liggins ${ }^{3}$ described successful pregnancy outcome in patients with lupus anticoagulant and fetal wastage who were treated with prednisone and low-dose aspirin. Prednisone and aspirin may improve fetal survival. However, it is important to remember that in the absence of clinical manifestations of lupus, prolonged use of high-dose prednisone may cause serious maternal side effects. The effects on the surviving infants are still unknown. ${ }^{8}$ Furthermore, recent data show that prednisone does not improve and may worsen fetal outcome in asymptomatic pregnant women with anticardiolipin antibody.

In our patients, low-dose aspirin therapy did prevent placental vascular thrombosis. We speculate that it may ameliorate the thrombotic episode and enable the fetus to reach the state of viability. The Wallenburg study suggests that the use of aspirin prevents fetal growth retardation in patients who are at risk for this complication. ${ }^{10}$ Trudinger et al $^{11}$ studied 44 patients with abnormal umbilical artery Doppler velocity waveforms during the third 


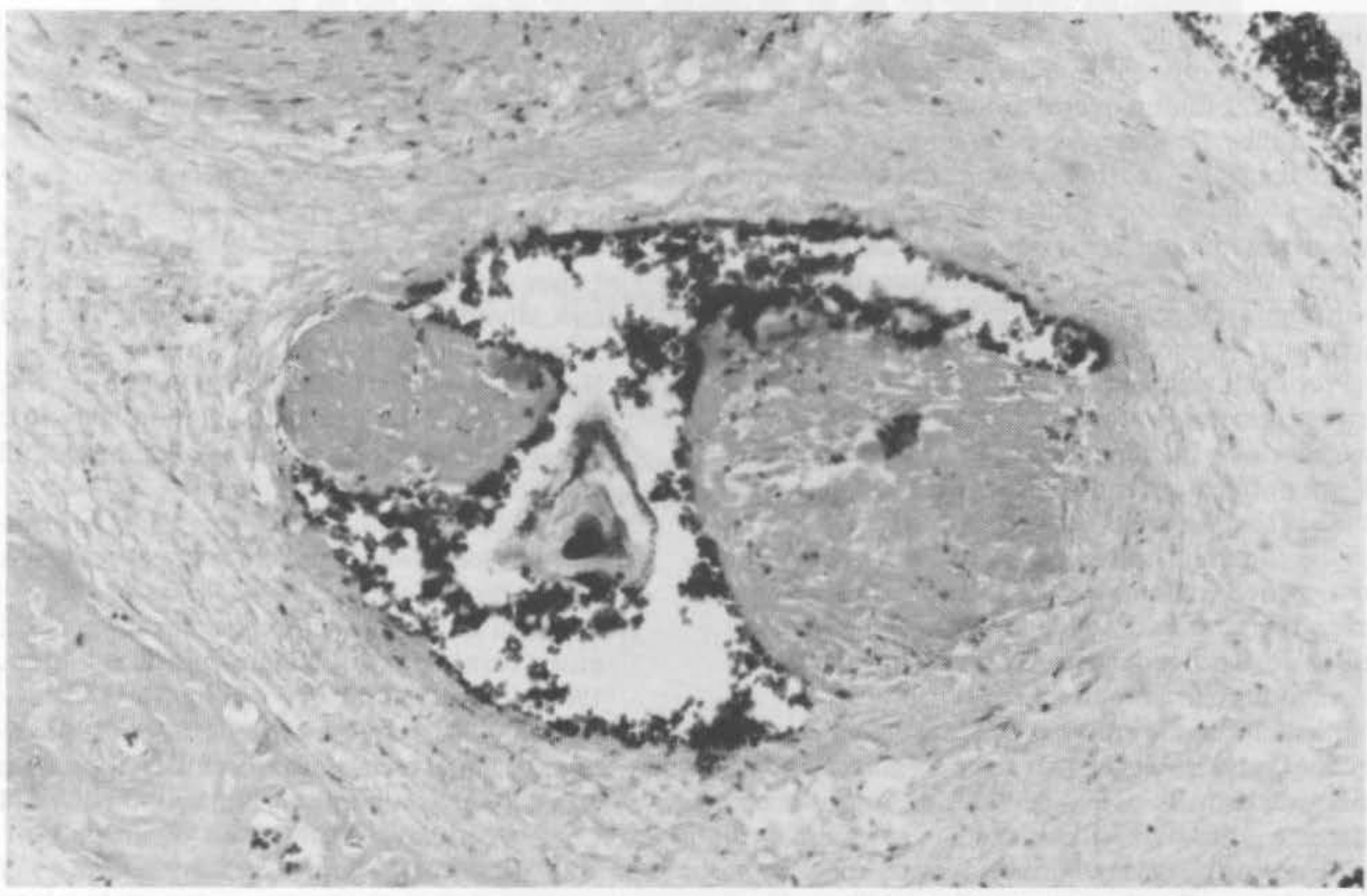

Figure 1. Subamniotic blood vessel containing thrombi (hematoxylin and eosin. (original magnification $\times$ $100)^{14}$

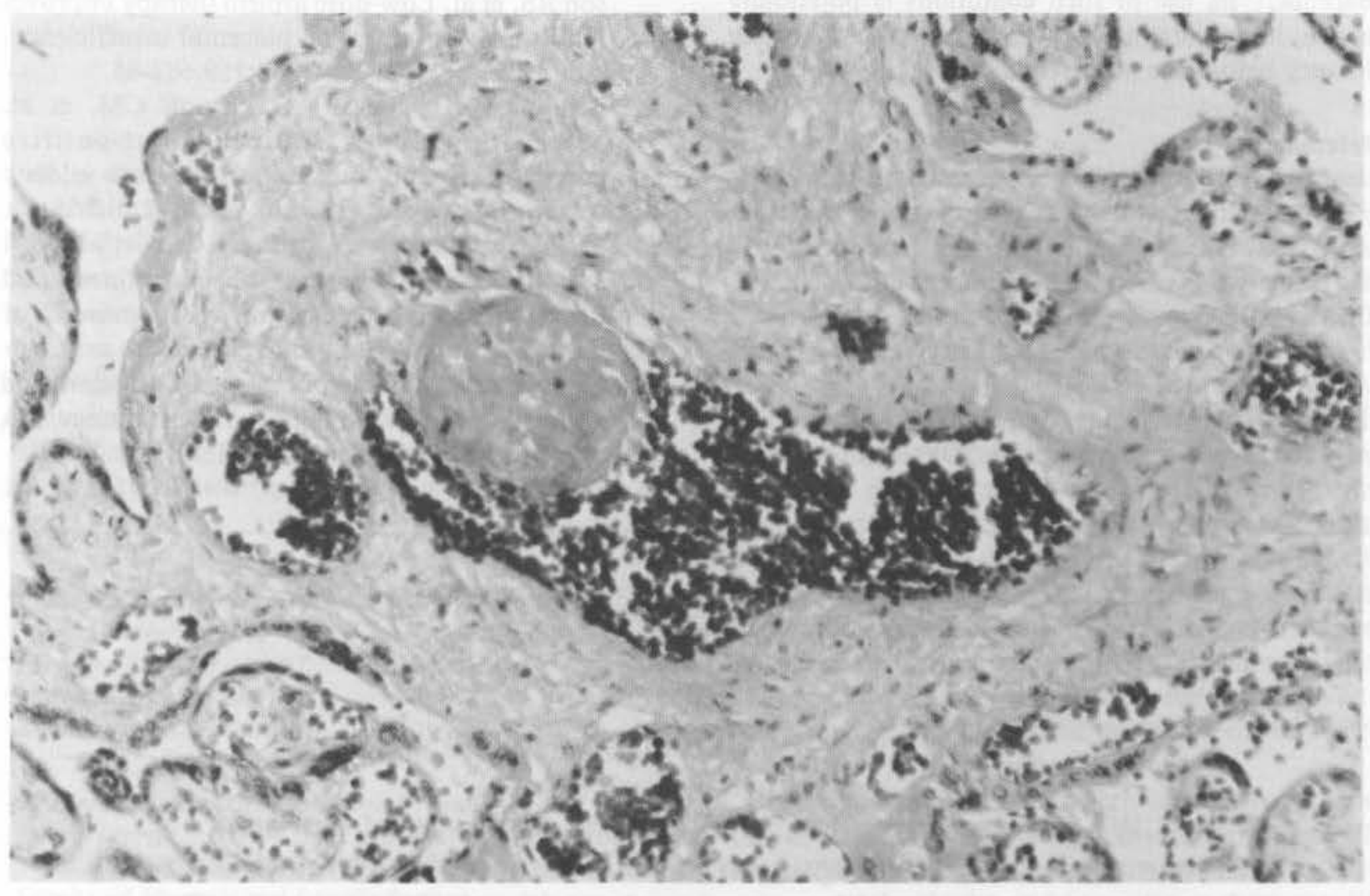

Figure 2. A stem chorionic villus with blood vessel containing recent thrombus (hematoxylin and eosin. (original magnification $\times 100)^{14}$ 
trimester of pregnancy. Twenty-one patients received $150 \mathrm{mg} /$ day of aspirin and the other 23 received placebo. The aspirin treated group had larger placentas and their fetuses weighed on an average $500 \mathrm{mg}$ more than the control group. ${ }^{11}$ It appears that therapy with low-dose aspirin may be useful in other conditions, but our study showed that it could not prevent placental vascular thrombosis in patients with lupus anticoagulant and/or anticrdiolipin antibodies.

Previous investigators have shown that umbilical artery velocity waveforms can reliably monitor pregnancies with lupus anticoagulant antibodies. ${ }^{12}$ We performed serial umbilical artery Doppler flow studies from 24 weeks onward. A progressive rise in systolic/diastolic ratio was noted throughout the study period without reversal of the diastolic component. The abnormal umbilical artery Doppler study led us to strict monitoring of these pregnancies in order to prevent intrauterine fetal demise.

In summary, we found that low-does aspirin therapy in presence of lupus anticoagulant and/or anticardiolipin antibodies does not prevent placental vascular thrombosis. However, it may have ameliorated the thrombotic episode and enabled the fetus to reach the stage of viability. Since systolic/diastolic ratio of the umbilical artery waveform provides a measure of impedance or resistance to blood flow downstream toward the placenta, ${ }^{13}$ its use in such conditions is potentially useful in predicting fetal compromise allowing delivery before the occurrence of fetal demise.

\section{References}

1. Lube TB, Bulter WS, Palmer SJ, Liggins GC. Lupus anticoagulant in pregnancy. $\mathrm{Br} \mathrm{J}$ Obstet Gynecol. 1984;91:357-63.

2. Branch DW, Scott JR, Kochenour NK, et al. Obstetric complications associated with the lupus

anti-coagulant. N Engl J Med.
1985;313:1322-26.

3. Lubbe WJ, Liggins GC. Lupus anticoagulant and pregnancy. Am J Obstet Gynecol.
$1985 ; 153: 322-7$.

4. Triplett DA, Brandt JT, Kaczor D, et al. Laboratory diagnosis of lupus inhabitor: A comparison of the tissue thromboplastin inhibitions procedure with a new platelet neutralization procedure. Am J Clin Path. 1983;678-82.

5. Gastineau DA, Kazmier FJ, Nichors WL, et al. Lupus anticoagulant: An analysis of the clinical and laboratory features of 219 cases. Am J Hematol. 1985;19:265-75.

6. Harris EN, Gharavi AE, Patel SP, Hughes GR. Evaluation of the anticardiolipin antibody test: Report of an international workshop held April 4, 1986. Clin Exp Immunol. 1987;68:215-22.

7. Schulman H, Fleischer A, Stern W, et al. Umbilical velocity wave ratios in human pregnancy. Am J Obstet Gynecol. 1984;148:985-90.

8. Feinstein DL. Lupus anticoagulant, thrombosis, and fetal loss. $N$ England J Med. 1985;313:1348-50.

9. Lockshin MD, Druzin ML, Qamar T. Prednisone does not prevent recurrent fetal death in women with antiphospholipid antibody. Am J Obstet Gynecol. 1989;160:439-43.

10. Wallenburg HC, Rotman N. Prevention of recurrent idiopathic fetal growth retardation by low-dose aspirin and dipyridamole. Am J Obstet Gynecol. 1987;157:1230.

11. Trudinger BJ, Stewart GJ, Cook CM, Thompson RS, et al. Low-dose aspirin therapy improves fetal weight in umbilical placental insufficiency. Am J Obstet Gynecol. 1988;159:681-85.

12. Trudinger BJ, Stewart GJ, Cook CM, et al. Monitoring lupus anticoagulant-positive pregnancies with umbilical artery flow velocity waveforms Obstet Gynecol. 1988;72:215-18.

13. Trudinger BJ, Giles WB, Cook CM, et al. Fetal umbilical artery flow velocity waveforms and placental resistance: Clinical significance. $\mathrm{Br} \mathrm{J}$ Obstet Gynecol. 1985;92:23-30.

14. Hadi HA, Treadwell E. Lupus anticoagulant and anticardiolipin antibodies in pregnancy. A Review Obstet Gynecol Surv (In press).

Part 1- - Part 2 